\title{
Archaeal cell biology: Diverse Functions of Tubulin-like Cytoskeletal Proteins at the Cell Envelope
}

Yan Liao, Solenne Ithurbide, Roshali T. de Silva, Susanne Erdmann, Iain G. Duggin

The ithree institute, University of Technology Sydney, Ultimo, NSW, 2007, Australia.

*Correspondence: Iain.Duggin@uts.edu.au 


\begin{abstract}
The tubulin superfamily is a widespread family of cytoskeletal proteins that appear in all three domains of life-Archaea, Bacteria and Eukarya. Tubulins build the microtubules of the eukaryotic cytoskeleton, whereas members of the homologous FtsZ family construct the division ring in prokaryotes and some eukaryotic organelles. Their functions are relatively poorly understood in archaea, yet these microbes contain a remarkable diversity of tubulinsuperfamily proteins, including FtsZ for division, a newly described major family called CetZ that is involved in archaeal cell shape control, and several other divergent families of unclear function that are implicated in a variety cell envelope remodelling contexts. Archaeal model organisms, particularly halophilic archaea such as Haloferax volcanii, have sufficiently developed genetic tools and we show why their large, flattened cells that are capable of controlled differentiation are also well suited to cell biological investigations by live-cell highresolution light and electron microscopy. As most archaea only have a glycoprotein lattice Slayer, rather than a peptidoglycan cell wall like bacteria, the activity of the tubulin-like cytoskeletal proteins at the cell envelope is expected to vary significantly, and may involve direct membrane remodelling or directed synthesis or insertion of the S-layer protein subunits. Further studies of archaeal cell biology will provide fresh insight into the evolution of cells and the principles in common to their fundamental activities across the full spectrum of cellular life.
\end{abstract}

\title{
Summary points
}

- Archaea contain homologs of bacterial FtsZ and eukaryotic tubulin cytoskeletal proteins, as well as other highly diverse members of this protein superfamily.

- Some archaea have CetZ family proteins, which control archaeal cell morphology and show similarities to both FtsZ and tubulin.

- Salt-loving Haloarchaea, particularly Haloferax volcanii, are well suited to studies of archaeal cell biology.

- Archaeal cell envelopes differ substantially compared to bacteria and eukaryotes; studies of cytoskeletal function will be valuable in identifying important principles retained amongst the most diverse cell types observed today, shedding light on the evolution of primordial cells. 


\section{Introduction}

Tubulins comprise a distinct family of GTPases that are the primary component of microtubules - one of the principal components of the eukaryotic cytoskeleton (Figure 1) [1, $2]$. The importance of tubulins in the course of early cellular evolution was realized with the discovery of its structural homology to prokaryotic cell division protein FtsZ, which forms the bacterial cytokinetic ring, despite the different cellular roles [3]. Recently, discovery of a function for proteins from a third related family in archaea, called CetZ, substantially expanded our knowledge of the breadth of diversity and functions these proteins carry out [4]. The tubulin, CetZ and FtsZ families, their sub-families and other related proteins are now recognised as members of the tubulin superfamily. These proteins are widely distributed across the three domains of life: Archaea, Bacteria, and Eukarya [5]. Archaea house some of the most diverse members of the tubulin superfamily including bacteria-like and eukaryotelike tubulin homologs, as well as family members specific for archaea [6].

Archaea represent a substantial proportion of global metabolic and genotypic diversity, alongside eukaryotes and bacteria, and are of major evolutionary, environmental and technological importance [7-9]. More recently, the importance of the archaea in cellular evolution has received considerable attention due to the identification of many genes in newly-identified archaeal phyla that encode proteins previously thought to be unique to eukaryotic cell biology, including tubulin [10]. The archaea also show apparently greater diversity amongst the most wide-spread cytoskeletal proteins found in all three domains, including diverse homologs from the actin and tubulin superfamilies. In the tubulin superfamily, archaea house a particularly diverse collection, including FtsZ in many of the major taxa, tubulin in some groups, as well as some archaea-specific families, some of which are widespread-others highly divergent [6]. Indeed, many archaea encode multiple tubulin superfamily homologs that have differing functions $[4,6]$. Remarkably, almost all members of the Crenarchaeota phylum do not possess a clear tubulin superfamily homolog and use alternative structural/scaffolding components, including a cell division apparatus based on homologs of the eukaryotic Endosomal Sorting Complex Required for transport (ESCRT)-III complex $[11,12]$. 
The availability of a rapidly increasing number of archaeal genomes is uncovering a remarkable diversity of tubulin superfamily proteins that will greatly expand their range of known functions across the domains of cellular life. When combined with functional studies of archaeal cells, these studies are expected to help unravel the evolutionary history of cells and the role of the cytoskeleton in early cellular evolution and diversification. As an example, the major archaeal protein family CetZ, which is related to both tubulin and FtsZ, was only recently characterized and shown to be wide-spread in the Euryarchaeaota phylum. CetZ1 exhibited a new role in cell shape regulation reminiscent of a genuine cytoskeletal function and might have existed in archaea before eukaryotic cell evolution [4]. In this review, we outline the CetZ and other families and subgroups of tubulin superfamily proteins in archaea, discussing functions and potential mechanisms of action for these proteins. We outline some of the recent and potential contributions that can be made by studying and developing model archaea that are suitable for cell biology studies and are already relatively established models of molecular biology and genetics.

\section{Haloarchaea as models for studying the cytoskeleton and cell biology}

Haloarchaea dominate hyper-saline lakes globally and are generally noted for their diverse cell shapes, including simple forms, such as rods [13], cocci [14] or disks [4], and more unusual forms, such as triangles [15], squares [16,17] or pleomorphic cells [18]. While it is appreciated that the cell envelope and surface layer of archaea are required for the cell's structural integrity, the molecular basis of cell shape and its regulation in haloarchaea is essentially unknown. The haloarchaea provide subjects that are well suited for studying the cytoskeleton and cell biology. For example, Halobacterium salinarum and Haloferax volcanii are readily cultured archaea that grow in high-salt heterotrophic media and both have quite welldeveloped genetic methods and tools available $[19,20]$. Both organisms show an remarkable plasticity of morphology, although in replete laboratory culture $H$. salinarum grow as rodshaped cells whereas $H$. volcanii are flattened plate-shaped cells [4, 21]. The flatness of $H$. volcanii and many other haloarchaea might relate to improving surface area and solute exchange in high salt environments [22]. $\mathrm{H}$. volcanii plate cells show moderate shape variations between individual cells and over time, but can conditionally develop into well- 
defined rod-shaped cells or other shape types that can include features such as irregular stalks and unusual elongated forms of variable width. In addition, the most motile cells of $H$. volcanii cultured in soft-agar medium are distinct rod-shaped cells of well-defined widths [4]. Thus, the controllable cellular differentiation $H$. volcanii was suggested as a model for understanding cytoskeletal function and morphological differentiation in archaea, which should also have broader implications for evolutionary cell biology [4].

For cell biological studies, we believe $H$. volcanii has some advantageous attributes. $H$. volcanii shows a stable genome, lower salt requirements, and arguably the most developed genetic "toolbox", with a range of efficient selectable genetic markers [23], improved shuttle/cloning vectors [24], reporter genes and fluorescent proteins $[4,25,26]$, constitutive/inducible promoters and protein production strains $[24,27,28]$, and efficient gene manipulation systems [19]. Its relatively large, flat cells are semi-transparent in light microscopy, and are thin enough for electron cryotomography. They do not produce gas vesicles that may complicate cell imaging, and they show no significant auto-fluorescence in the visible spectrum, despite the presence of carotenoid pigments in the cell membranes of these organisms [4]. Fluorescence-based (DNA staining) flow- and Coulter-cytometry are also established for $H$. volcanii, allowing sensitive measurements of cell cycle, size and division parameters $[4,29]$. Combining these features and the above tools with high- or superresolution, live-cell and time-lapse microscopy to analyse the regulated cell differentiation responses of $H$. volcanii, has created a powerful system for the study of cytoskeletal elements and cell biology of the archaea (Figure 2). H. volcanii and other archaea contain multiple members of the tubulin superfamily (Table 1), many of which form conserved orthologous groups that are wide-spread across the archaeal domain or beyond [4, 30-32].

\section{FtsZ family proteins in archaea}

FtsZ proteins are tubulin-superfamily proteins that play a fundamental role in forming a contractile ring for division of prokaryotic cells (bacteria and archaea), or organelle division in some eukaryotes. FtsZ proteins form a distinct family with overall moderate sequence similarities with eukaryotic tubulins, but share the same overall structure and GTPase activesite features [33]. In addition to the globular domain structure of proteins of the tubulin 
superfamily, the FtsZ proteins frequently have characteristic $\mathrm{N}$ - and $\mathrm{C}$ - terminal extensions [3] that in known cases function in mediating interactions with other molecules required for cell division, such as membrane binding adapters or polymerization modulating proteins [34].

Studies of archaeal FtsZ proteins were initiated prior to the availability of whole genome sequences, and focussed on the identification and basic sub-cellular localization of an FtsZ homolog in a few species of archaea. Biochemistry and Immuno-fluorescence microscopy studies indicated that archaeal FtsZ proteins are GTPases and localized to the mid-cell plane of division, much like their bacterial counterpart [30-32,35]. Whereas most bacterial species studied so far contain only one FtsZ, many archaea are now known to carry two FtsZ homologues, one from each of two distinct sub-families, with FtsZ1 being apparently more closely related to bacterial and plant FtsZ, with FtsZ2 being more phylogenetically distinct [5, $6,36]$. The early studies had identified only the FtsZ1 homolog but not the FtsZ2 homolog that was also present in the archaea chosen for study $[32,35]$. One exception was the FtsZ2 homologue studied in $H$. salinarum [6, 30], which appeared to cause altered cell morphology after its overexpression in $\mathrm{H}$. salinarum [30].

The two distinct archaeal FtsZ subfamilies are constituted by members from diverse archaeal taxa, indicating that in each organism the two FtsZ proteins would be expected have differing functions or activities, both important to archaeal cell survival. The single bacterial FtsZ acts as scaffolding for protein recruitment to the division site [37], and can also effect membrane constriction [38,39], and guide cell wall synthesis to build the division septum [40,41]. Thus, it is of interest to determine how the function(s) of the two FtsZ proteins in archaea relate to the multiple functions of bacterial FtsZ. Interestingly, some archaea from deeply-rooted branches of the archaeal tree have only one specific FtsZ-family homolog [6], suggesting that the duplication generating the FtsZ1 and FtsZ2 archaeal families may have occurred after the diversification of the major bacterial and archaeal lineages (prior to the evolution of eukaryotes). One might expect that these solo archaeal FtsZ proteins have retained, in common with bacteria, some of the most fundamental features of FtsZ activity that first evolved in the tubulin superfamily. As will be discussed further below, archaeal cell envelopes differ substantially from bacterial envelopes, including the absence of a covalent cell wall 
structure, arguing that directing bacterial peptidoglycan synthesis was not the first-nor currently the only-function of FtsZ in division constriction.

The role of FtsZ in archaeal cell division was demonstrated genetically only recently. Expression of GTPase-defective FtsZ1 point mutant (D250A) in $H$. volcanii resulted in a dominant cell division defect, producing giant plate cells $[4,6]$. This also suggests that the GTPase activity of FtsZ1 is required for cell division. In the same study, FtsZ1 was tagged with GFP, which showed mid-cell localization in live $H$. volcanii [4] that was consistent with previous immuno-fluorescence studies [31, 32]. The presence of FtsZ1-GFP at a low level in a wild-type genetic background resulted in cells of normal size, indicating minimal or no significant disturbance to division by FtsZ1-GFP. Both the plate and rod cell types expressing FtsZ1-GFP showed mid-cell contractile rings or partial/split rings, generally similar to bacterial FtsZ-rings. Interestingly, almost all of the $H$. volcanii cells expressing low levels of FtsZ1-GFP in cultures exhibited rings, most without visible constriction, indicating $H$. volcanii FtsZ1 is capable of localizing for "Z-ring" formation at a very early stage of the division cycle, far in advance of visible mid-cell constriction [4]. In bacteria, the timing of Z-ring assembly in the division cycle can also occur in newborn cells immediately after the preceeding division or may occur later in the cell cycle depending on conditions and growth rate $[42,43]$, but it still occurs well in advance of the onset of division constriction to allow time for proper assembly of the functional divisome $[42,44,45]$.

It will be informative to observe the function and timing of assembly of FtsZ in other archaea, and at different growth rates/conditions, in order to compare the general features of archaeal versus bacterial FtsZ function in division. Compared to different bacterial models, archaeal systems offer a most diverse comparison with the well known bacterial FtsZ-based division systems, and will provide important evolutionary information that will allow identification of the most conserved and important features of FtsZ function across the full spectrum of prokaryotic life. 


\section{The CetZ family in archaea}

Archaeal CetZ proteins were characterized only recently and form an archaea-specific branch that is related to but clearly distinct from the tubulin and FtsZ families [4, 6, 36] (Figure 3). The primary sequences show various similarities to both tubulins and FtsZ proteins, leaving the evolutionary pathway of CetZ compared to the major tublin and FtsZ groups mysterious. For example, key highly conserved catalytic residues show matches with tubulin but not FtsZ, while the C-terminal domain shows somewhat greater sequence similarity to FtsZ proteins. CetZ genes are common in haloarchaea, but are also found in a wide variety of genomes from across the phylum Euryarchaeota, including Classes Methanomicrobia and Thermococci [6]. For example, six homologues are present in $H$. volcanii, but the number varies among different species. Crystal structures of $\mathrm{H}$. volcanii CetZ1, CetZ2 (Figure 3b) and Methanosaeta thermophila CetZ showed a typical FtsZ/tubulin fold for GTP binding and hydrolysis. As shown in Figure 3b, the two core domains of the globular fold are an N-terminal GTP-binding domain and a more divergent C-terminal domain involved in self-association, separated by a central helix and loop carrying catalytic residues responsible for hydrolysis of GTP in the neighbouring subunit in a polymer. A C-terminal $\alpha$-helical extension shows some structural similarities to the tubulin C-terminal extension. Also like tubulin, CetZ proteins do not typically contain the $\mathrm{N}$-terminal extension seen in FtsZ [4] (Figure 3a). H. volcanii CetZ2 crystallized as 2D sheets of protofilaments showing both longitudinal and lateral interactions like tubulin in the microtubule wall, implying a feasible cytoskeletal or cytomotive function [4].

The overall CetZ family can be resolved into distinct orthologous groups amongst haloarchaea. The group containing CetZ1 is the most conserved, with approximately $80 \%$ sequence identity seen amongst homologs from other members of the Class Halobacteria, followed by the group containing CetZ2. H. volcanii CetZ1 and CetZ2 are the only two proteins from the greater CetZ family that have function ascribed - in controlling cell shape [4]. $H$. volcanii CetZ3-6 are more diverse, whilst many haloarchaea that contain CetZ family proteins have only clear homologs of CetZ1 or possibly CetZ2 [6]. Archaea that have CetZ were also noted to contain at least one additional FtsZ protein [6]. 
Genetic approaches were first used to establish a function for the prototypical CetZ protein, CetZ1, from H. volcanii [4]. Individual deletion of the six CetZs, or the expression of dominantinhibitory mutants in $\mathrm{H}$. volcanii had not revealed an effect on the growth rate or cell size, suggesting that the CetZ's are not required for cell division. However, in soft-agar motility assays, deletion of CetZ1 prevented the differentiation of the irregular plate-shaped cells into a rod-shaped cell type required for normal swimming motility. Furthermore, overproduction of CetZ1 stimulated the development of rod-like morphology in plate cells [4].

Expression of a CetZ1-GFP fusion protein showed patchy or diffuse fluorescence in $\mathrm{H}$. volcanii, usually around the cell periphery or at mid-cell. This pattern was highly dynamic in live cells, with localizations moving over timeframes of several minutes, which was dependent on the integrity of the GTPase active site [4]. Indeed mutation of a key catalytic residue (E218) to prevent GTPase activity and inhibit the de-polymerization cycle, created hyper-stable localizations at particular locations apparently at or near the cell envelope that resulted in unusual cell surface shapes and structures, including short stalks and sharp inflections in the cell envelope. During rod development, expression of wild-type CetZ1-GFP showed evidence of faint, dynamic filaments that were often aligned with the long axis of the cell, suggesting that they may be directly relevant to the rod-development process, through as yet unknown mechanisms. These appear to differ from the patterns observed for bacterial proteins involved in rod development, notably membrane-associated MreB that orients along the greatest principal membrane curvature to guide cell wall synthesis to create rod shape [46].

CetZ2 of $\mathrm{H}$. volcanii was also implicated in cell shape control, however deletion of CetZ2 or its overproduction did not result in the phenotypes seen with CetZ1, and they appeared the same as in the wild-type strain. Instead, the cell shape effects were observed during expression of GTPase-defective mutant CetZ2 (CetZ2.E212A) in wild-type background inhibited motility and rod development in a manner that was dominant over the wild-type CetZ2 in these cells, and this mutant also resulted in a similar "jagged" cell shape phenotype as seen for CetZ1.E218A. These data indicated that CetZ2 is likely to have a role in controlling cell shape either in conjunction with (and at least sometimes redundant to) CetZ1, or in response to other environmental conditions or different purposes. 
Cytoskeletal proteins often have multiple functions, and are adapted for use in various biological contexts. A recent study analysing the composition of membrane vesicles (MVs) of a haloarchaeal species, Halorubrum lacusprofundi, revealed the presence of a CetZ1 homolog in MVs and specialised plasmid-containing membrane vesicles (PVs) [47]. This protein was significantly more enriched in purified vesicles when compared with membrane fractions prepared from the host, suggesting that it might play a role in membrane vesicle formation or structure [47]. Precedent for this type of activity comes from studies of Crenarchaeota that lack tubulin superfamily proteins, where the ESCRT-III (CdvB) proteins were reported to play a role in $\mathrm{MV}$ formation and in division [11, 12, 48, 49]. FtsZ proteins have also been detected in bacterial MVs $[50,51]$ although it is unclear if this is directly related to their generation. Finally, it is possible that CetZ or other tubulin-like proteins in archaea participate in DNA segregation or cellular fusion and mating, by analogy to the roles of tubulin and certain bacteriophage homologs [52]. Tubulins were shown to play a crucial role for mating in unicellular Eukarya $[53,54]$. Mating and cell fusion is a common mechanism for gene exchange in haloarchaea [55]. It is therefore possible that CetZ or other tubulin-like proteins play roles in DNA segregation, however, these potential roles remain to be elucidated.

\section{The tubulin family in archaea}

Specific tubulin family proteins (not to be confused with the broarder tubulin superfamily that includes FtsZ and CetZ) are rare in prokaryotes. However, in recent years tubulin family homologs have been identified in cetain archaea and bacteria, evoking much interest in their origins. Genes encoding distant relatives of the tubulin family, called artubulins, were found in genomes of two species of Thaumarchaeota (from the genus Nitrosoarchaeum) [56]. Several other artubulin homologs have been sequenced more recently in closely related Thaumarchaeota (Candidatus genera, Nitrosotenuis and Nitrosotalea), but have not yet been identified more widely in other archaea. The Artubulins stem from the base of the tubulin family, forming a sister group to the eukaryotic tubulin family [56]. This raises the possibility that artubulins could be the direct ancestors of tubulins, suggesting that the tubultin family originated in archaea before the divergent evolution of the eukaryotic cell lineage [56]. Alternatively, their limited distribution in certain Thaumarchaeota suggests that they might 
have been retained or acquired by the Nitrosoarchaeum lineage after the split that led to the divergence of eukaryotes and archaea. It will therefore be of interest to conduct genomic analyses that investigate the potential for ancient horizontal gene transfer from eukaryotes, as well as structural and functional studies of the proteins, for comparison with eukaryotic tubulins. Although their functions are currently unknown, the genomic proximity of the artubulin genes to a snf7 gene implicated in ESCRT-III mediated division raises the hypothesis that artubulins might cooperate with ESCRT-III and function in division or membrane remodelling processes in these organisms [56-58].

Two archaeal tubulin family proteins were also recently detected in metagenomic sequence data in the candidate phylum Odinarchaeota [10]. These organisms belong to the "Asgard" superphylum, which is a recently described group of archaea that affiliate with eukaryotes in phylogenomic analyses and show a remarkably high number of other proteins that were previously thought to be unique to eukaryotes, including apparent organelle trafficking and intracellular signalling systems [10]. Phylogenetic analyses identified the Asgard archaea tubulins as another sister group to eukaryotic tubulins, distinct from artubulins [10]. Their existance lends some support to the idea that the tubulin family arose in early archaea-like cells and then diversified extensively during the evolution eukaryotic cells, yet has only been retained in a few modern-day archaea. Given their greater similarity to eukaryotic tubulins, compared to artubulins, there is expected to be considerable interest in determining the filament structures, subcellular localization and cellular functions of the Asgard and Thaumarchaeota tubulins. Ideally, such studies would utilize isolated strains of these currently uncultured archaea, or may employ surrogate or even synthetic (Asgard-like) organisms in future.

\section{Other diverse tubulin-superfamily homologs in archaea}

The phylum Thaumarchaeota shows a particularly complex distribution of tubulin superfamily members amongst the various taxa. There are also Thaumarchaeota-specific groups, including the unusual "Thaumarchaeota-FtsZ" family, which shows only some of the tubulin/FtsZ signature sequences and lacks several key catalytic regions required for GTP hydrolysis. Consistent with this, the Thaumarchaeota-FtsZ protein in Nitrosopumilis 
maritimus was observed diffuse throughout these cells and not polymerized at division sites, whereas the CdvB (ESCRT-III) homolog localized to mid-cell sites and was implicated as the primary cell division protein instead [59]. Furthermore, the Thaumarchaeota-FtsZ protein was diffuse when expressed in yeast and only showed localization consistent with polymerization when the E. coli T4 loop (for GTP binding) was substituted into the protein [60]. It is therefore unclear whether these proteins can normally polymerize or what their functions could be, but answers will likely await the development of genetic analysis methods for Thaumarchaeota.

Two highly atypical "FtsZ-like" protein families (FtsZL1 and FtsZL2) have also been identified in prokaryotes (bacteria and archaea), and these proteins share homology to only the core GTP-binding domain of the tubulin superfamily, and contain additional large domains of unknown function [61]. FtsZL2 is only found in Proteobacteria, but FtsZL1 shows a scattered distribution across a few bacterial and Euryarchaeota groups, with the archaeal ones forming a phylogenetic subgroup distinct from the related bacterial ones [61]. FtsZL1 proteins are also found sporadically in other archaeal phyla, notably including a specific sub-group with an extra N-terminal domain found in the Crenarchaeota, which contain no other tubulin superfamily members [6]. FtsZL1 proteins generally lack several of the key structural elements required for GTP-hydrolysis and polymerization, and therefore are not expected to form FtsZ/tubulin-like filaments [61]. FtsZL1 genes are associated with a set of genes that are predicted to be secreted or membrane associated hinting at a possible role involving membrane remodelling [61], but are currently of unknown function.

Highly diverse tubulin superfamily proteins also appear in archaea that have highly unusual cell shapes; for example, the strikingly "square" cells of Haloquadratum walsbyi [4]. H. walsbyi is known to form postage stamp-like sheets when cells do not separate after division [62]. It is difficult to study the function of these highly-divergent tubulin-superfamily members because genetic systems have not been developed for $\mathrm{H}$. walsbyi, but we speculate that the numerous tubulin superfamily proteins may function in maintaining the square shape, which itself is of unclear function.

\section{How might tubulin superfamily proteins work in archaea?}


By using the FtsZ proteins as an example, we now discuss possible similarities and differences between the mechanisms that could exist in the division of bacterial and archaeal cells. While the components and envelope-remodelling outcomes may vary, the basic principles are likely to be retained by other archaeal tubulin-like proteins, such as CetZ.

In almost all bacteria, FtsZ is among the first molecules to localize at the division site to assembly the contractile "Z-ring" that acts as a scaffold for the recruitment of the other components of the divisome required for cytokinesis and cell wall remodelling to build the transient septum between the dividing cells (Figure 4) [63]. In many Gram-negative bacteria such as E. coli, FtsZ protofilaments are anchored to the membrane at the division site through actin-like ATPase protein FtsA and ZipA and are stabilized and regulated by Z-ring associated proteins (ZapA-D) and other spatial and cell cycle regulators [34]. Some of these are conserved in Gram-positive bacteria, which also contain other proteins of related function, including a FtsZ membrane anchoring protein, SepF [64]. Interestingly, almost no archaeal genomes have been found to encode distinct homologs of FtsA or ZipA, but homologs of SepF have been found in FtsZ-containing archaea [5, 58], suggesting that a FtsZ-SepF complex might play a similar role for cell division in archaea.

In addition to providing a scaffolding-like assembly function for the division ring, FtsZ plays an active part in the membrane constriction and cell wall ingrowth processes during bacterial division [38-41]. FtsZ filaments undergo dynamic treadmilling polymerization around the ring at the inner surface of the cell membrane during constriction, actively directing the activities of the peptidoglycan cell wall synthesis machinery linked to the outside of the membrane, thus leading to wall ingrowth and eventual closure of the septum [40,41]. While it is anticipated that this dynamic polymerization pattern occurs in FtsZ-containing archaea [4, 32], it is interesting to consider how this may contribute to division, given the very different envelope architecture of archaea and bacteria.

Most archaea are not predicted to contain cell walls consisting of a covalent 3D-mesh, like the peptidoglycan-based walls of bacteria, which are expected to be conducive to remodelling via the spatially-regulated addition or removal of small molecular subunits. Instead, archaea typically only have a single membrane plus an outer S-layer composed of non-covalent arrays 
(typically hexagonal) of a membrane anchored S-layer glycoprotein (SGP) [65]. (see Figures $2 \mathrm{~b}$ and $3 \mathrm{c})$. The membrane lipids of archaea are also different, and are primarily composed of glycerol-1-phosphate ether lipids with branched isoprenoid chains, in contrast to bacterial/eukaryotic membranes based on glycerol-3-phosphate ester lipids and fatty acid chains.

Whether archaeal FtsZ polymerization directs the SGP synthetic machinery at the division site to localize the insertion of new SGP is a key question. However, the non-covalent and possibly more flexible 2D crystalline structure containing very high molecular mass S-layer glycoprotein subunits in archaea means that the underlying mechanism would be necessarily very different compared to bacteria. Pioneering electron microscopy studies on the S-layer in FtsZ-containing archaea revealed disruptions in the lattice symmetry at the division furrow through various stages of invagination [66]. Based on 2D crystal lattice theory, these disruptions are consistent with the insertion of new morphological units at these sites [66]. In addition, the S-layer subunits would need to pivot substantially with the strong curvature experienced during invagination, and then separate completely, resealing to form a uninterrupted envelope in each new cell. Insertion of new SGP at the division site by an FtsZdirected mechanism would be a plausible mechanism to allow this process, and could also seal any gaps remaining after closure. Finally, the mono-layer nature of the archaeal S-layer, and its significant flexibility that gives rise to a remarkable cell malleability [4, 21], creates an additional challenge for FtsZ-based division: how to ensure that division proceeds inwards, when SGP insertion alone would not be expected to cause inward growth, like PG built inwards on a 3D mesh could in bacteria. We therefore predict that inward growth in archaeal division requires a direct lipid-membrane constriction activity of the archaeal divisome or FtsZ, as has been directly observed with purified bacterial FtsZ anchored to membrane liposomes $[38,39]$.

Researchers now have the tools and model organisms that have in recent years begun to reveal the functions of some of the most conserved proteins identified in archaea based on homology to well-studied bacterial and eukaryotic counterparts. But this represents only a tiny fraction of the myriad of components for cell biological functions and mechanisms that 
archaea must carry out. For example, the majority of the 30 or more known bacterial division proteins have not been clearly identified amongst the FtsZ-containing archaea, suggesting that very different mechanisms based on the core tubulin superfamily proteins exist in archaea compared to bacteria. This also argues that the divisome and cell biological complexity would have been very primitive during the time of the evolutionary divergence of bacteria and archaea. Accordingly, we expect that an understanding of archaeal FtsZ-based division and other cytoskeletal functions will be of great value in shedding new light on the fundamental principles that govern cell division and cytoskeletal protein function across the three domains of life. 


\section{References}

1 Nogales, E. (2000) Structural insights into microtubule function. Annu Rev Biochem. 69, 277-302

2 Wade, R. H. (2009) On and around microtubules: an overview. Mol Biotechnol. 43, 177-191

3 Lowe, J. and Amos, L. A. (1998) Crystal structure of the bacterial cell-division protein FtsZ. Nature. 391, 203-206

4 Duggin, I. G., Aylett, C. H., Walsh, J. C., Michie, K. A., Wang, Q., Turnbull, L., Dawson, E. M., Harry, E. J., Whitchurch, C. B., Amos, L. A. and Lowe, J. (2015) CetZ tubulin-like proteins control archaeal cell shape. Nature. 519, 362-365

$5 \quad$ Wagstaff, J. and Lowe, J. (2018) Prokaryotic cytoskeletons: protein filaments organizing small cells. Nat Rev Microbiol. 16, 187-201

6 Aylett, C. H. S. and Duggin, I. G. (2017) The Tubulin Superfamily in Archaea. Subcell Biochem. 84, 393-417

7 DeLong, E. F. and Pace, N. R. (2001) Environmental diversity of bacteria and archaea. Syst Biol. 50, 470-478

8 Schiraldi, C., Giuliano, M. and De Rosa, M. (2002) Perspectives on biotechnological applications of archaea. Archaea. 1, 75-86

9 Woese, C. R., Kandler, O. and Wheelis, M. L. (1990) Towards a natural system of organisms: proposal for the domains Archaea, Bacteria, and Eucarya. Proc Natl Acad Sci U S A. 87, 4576-4579

10 Zaremba-Niedzwiedzka, K., Caceres, E. F., Saw, J. H., Backstrom, D., Juzokaite, L., Vancaester, E., Seitz, K. W., Anantharaman, K., Starnawski, P., Kjeldsen, K. U., Stott, M. B., Nunoura, T., Banfield, J. F., Schramm, A., Baker, B. J., Spang, A. and Ettema, T. J. (2017) Asgard archaea illuminate the origin of eukaryotic cellular complexity. Nature. 541, 353-358

11 Lindas, A. C., Karlsson, E. A., Lindgren, M. T., Ettema, T. J. and Bernander, R. (2008) A unique cell division machinery in the Archaea. Proc Natl Acad Sci U S A. 105, 18942-18946

12 Samson, R. Y., Obita, T., Freund, S. M., Williams, R. L. and Bell, S. D. (2008) A role for the ESCRT system in cell division in archaea. Science. 322,1710-1713

13 Mormile, M. R., Biesen, M. A., Gutierrez, M. C., Ventosa, A., Pavlovich, J. B., Onstott, T. C. and Fredrickson, J. K. (2003) Isolation of Halobacterium salinarum retrieved directly from halite brine inclusions. Environ Microbiol. 5, 1094-1102

14 Montero, C. G., Ventosa, A., Rodriguez-Valera, F., Kates, M., Moldoveanu, N. and Ruiz-Berraquero, F. (1989) Halococcus saccharolyticus sp. nov., a new species of extremely halophilic non-alkaliphilic cocci. Systematic and applied microbiology. 12, 167-171

15 Takashina, T., Hamamoto, T., Otozai, K., Grant, W. D. and Horikoshi, K. (1990) Haloarcula japonica sp. nov., a new triangular halophilic archaebacterium. Systematic and Applied Microbiology. 13, 177-181

16 Burns, D. G., Janssen, P. H., Itoh, T., Kamekura, M., Li, Z., Jensen, G., RodriguezValera, F., Bolhuis, H. and Dyall-Smith, M. L. (2007) Haloquadratum walsbyi gen. nov., sp. nov., the square haloarchaeon of Walsby, isolated from saltern crystallizers in Australia and Spain. Int J Syst Evol Microbiol. 57, 387-392

17 Walsby, A. E. (1980) A square bacterium. Nature. 283, 69 
18 Mullakhanbhai, M. F. and Larsen, H. (1975) Halobacterium volcanii spec. nov., a Dead Sea halobacterium with a moderate salt requirement. Arch Microbiol. 104, 207214

19 Allers, T. and Mevarech, M. (2005) Archaeal genetics - the third way. Nat Rev Genet. 6, 58-73

20 Silva-Rocha, R., Pontelli, M. C., Furtado, G. P., Zaramela, L. S. and Koide, T. (2015) Development of New Modular Genetic Tools for Engineering the Halophilic Archaeon Halobacterium salinarum. PLoS One. 10, e0129215

21 Eun, Y. J., Ho, P. Y., Kim, M., LaRussa, S., Robert, L., Renner, L. D., Schmid, A., Garner, E. and Amir, A. (2018) Archaeal cells share common size control with bacteria despite noisier growth and division. Nat Microbiol. 3, 148-154

22 Fendrihan, S., Legat, A., Pfaffenhuemer, M., Gruber, C., Weidler, G., Gerbl, F. and Stan-Lotter, H. (2006) Extremely halophilic archaea and the issue of long-term microbial survival. Rev Environ Sci Biotechnol. 5, 203-218

23 Allers, T., Ngo, H. P., Mevarech, M. and Lloyd, R. G. (2004) Development of additional selectable markers for the halophilic archaeon Haloferax volcanii based on the leuB and trpA genes. Appl Environ Microbiol. 70, 943-953

24 Allers, T., Barak, S., Liddell, S., Wardell, K. and Mevarech, M. (2010) Improved strains and plasmid vectors for conditional overexpression of His-tagged proteins in Haloferax volcanii. Appl Environ Microbiol. 76, 1759-1769

25 Holmes, M. L. and Dyall-Smith, M. L. (2000) Sequence and expression of a halobacterial beta-galactosidase gene. Mol Microbiol. 36, 114-122

26 Reuter, C. J. and Maupin-Furlow, J. A. (2004) Analysis of proteasome-dependent proteolysis in Haloferax volcanii cells, using short-lived green fluorescent proteins. Appl Environ Microbiol. 70, 7530-7538

27 Large, A., Stamme, C., Lange, C., Duan, Z., Allers, T., Soppa, J. and Lund, P. A. (2007) Characterization of a tightly controlled promoter of the halophilic archaeon Haloferax volcanii and its use in the analysis of the essential cct1 gene. Mol Microbiol. 66, 1092-1106

28 Strillinger, E., Grotzinger, S. W., Allers, T., Eppinger, J. and Weuster-Botz, D. (2016) Production of halophilic proteins using Haloferax volcanii H1895 in a stirredtank bioreactor. Appl Microbiol Biotechnol. 100, 1183-1195

29 Delmas, S., Duggin, I. G. and Allers, T. (2013) DNA damage induces nucleoid compaction via the Mre11-Rad50 complex in the archaeon Haloferax volcanii. Mol Microbiol. 87, 168-179

30 Margolin, W., Wang, R. and Kumar, M. (1996) Isolation of an ftsZ homolog from the archaebacterium Halobacterium salinarium: implications for the evolution of FtsZ and tubulin. J Bacteriol. 178, 1320-1327

31 Poplawski, A., Gullbrand, B. and Bernander, R. (2000) The ftsZ gene of Haloferax mediterranei: sequence, conserved gene order, and visualization of the FtsZ ring. Gene. 242, 357-367

32 Wang, X. and Lutkenhaus, J. (1996) FtsZ ring: the eubacterial division apparatus conserved in archaebacteria. Mol Microbiol. 21, 313-319

33 Nogales, E., Downing, K. H., Amos, L. A. and Lowe, J. (1998) Tubulin and FtsZ form a distinct family of GTPases. Nat Struct Biol. 5, 451-458

34 Ortiz, C., Natale, P., Cueto, L. and Vicente, M. (2016) The keepers of the ring: regulators of FtsZ assembly. FEMS Microbiol Rev. 40, 57-67 
35 Baumann, P. and Jackson, S. P. (1996) An archaebacterial homologue of the essential eubacterial cell division protein FtsZ. Proc Natl Acad Sci U S A. 93, 6726-6730

36 Vaughan, S., Wickstead, B., Gull, K. and Addinall, S. G. (2004) Molecular evolution of FtsZ protein sequences encoded within the genomes of archaea, bacteria, and eukaryota. J Mol Evol. 58, 19-29

37 Adams, D. W. and Errington, J. (2009) Bacterial cell division: assembly, maintenance and disassembly of the Z ring. Nat Rev Microbiol. 7, 642-653

38 Osawa, M. and Erickson, H. P. (2013) Liposome division by a simple bacterial division machinery. Proc Natl Acad Sci U S A. 110, 11000-11004

39 Szwedziak, P., Wang, Q., Bharat, T. A., Tsim, M. and Lowe, J. (2014) Architecture of the ring formed by the tubulin homologue FtsZ in bacterial cell division. Elife. 3 , e04601

40 Bisson-Filho, A. W., Hsu, Y. P., Squyres, G. R., Kuru, E., Wu, F., Jukes, C., Sun, Y., Dekker, C., Holden, S., VanNieuwenhze, M. S., Brun, Y. V. and Garner, E. C. (2017) Treadmilling by FtsZ filaments drives peptidoglycan synthesis and bacterial cell division. Science. 355, 739-743

41 Yang, X., Lyu, Z., Miguel, A., McQuillen, R., Huang, K. C. and Xiao, J. (2017) GTPase activity-coupled treadmilling of the bacterial tubulin FtsZ organizes septal cell wall synthesis. Science. 355, 744-747

42 Sun, Q. and Margolin, W. (1998) FtsZ dynamics during the division cycle of live Escherichia coli cells. J Bacteriol. 180, 2050-2056

43 Weart, R. B. and Levin, P. A. (2003) Growth rate-dependent regulation of medial FtsZ ring formation. J Bacteriol. 185, 2826-2834

44 Gamba, P., Veening, J. W., Saunders, N. J., Hamoen, L. W. and Daniel, R. A. (2009) Two-step assembly dynamics of the Bacillus subtilis divisome. J Bacteriol. 191, 41864194

45 Goley, E. D., Yeh, Y. C., Hong, S. H., Fero, M. J., Abeliuk, E., McAdams, H. H. and Shapiro, L. (2011) Assembly of the Caulobacter cell division machine. Mol Microbiol. 80, 1680-1698

46 Hussain, S., Wivagg, C. N., Szwedziak, P., Wong, F., Schaefer, K., Izore, T., Renner, L. D., Holmes, M. J., Sun, Y., Bisson-Filho, A. W., Walker, S., Amir, A., Lowe, J. and Garner, E. C. (2018) MreB filaments align along greatest principal membrane curvature to orient cell wall synthesis. Elife. 7

47 Erdmann, S., Tschitschko, B., Zhong, L., Raftery, M. J. and Cavicchioli, R. (2017) A plasmid from an Antarctic haloarchaeon uses specialized membrane vesicles to disseminate and infect plasmid-free cells. Nat Microbiol. 2, 1446-1455

48 Ellen, A. F., Albers, S. V., Huibers, W., Pitcher, A., Hobel, C. F., Schwarz, H., Folea, M., Schouten, S., Boekema, E. J., Poolman, B. and Driessen, A. J. (2009) Proteomic analysis of secreted membrane vesicles of archaeal Sulfolobus species reveals the presence of endosome sorting complex components. Extremophiles. 13, 67-79

49 Liu, J., Gao, R., Li, C., Ni, J., Yang, Z., Zhang, Q., Chen, H. and Shen, Y. (2017) Functional assignment of multiple ESCRT-III homologs in cell division and budding in Sulfolobus islandicus. Mol Microbiol. 105, 540-553

50 Aguilera, L., Toloza, L., Gimenez, R., Odena, A., Oliveira, E., Aguilar, J., Badia, J. and Baldoma, L. (2014) Proteomic analysis of outer membrane vesicles from the probiotic strain Escherichia coli Nissle 1917. Proteomics. 14, 222-229

51 Kim, Y., Edwards, N. and Fenselau, C. (2016) Extracellular vesicle proteomes 
reflect developmental phases of Bacillus subtilis. Clin Proteomics. 13, 6

52 Bertozzi Silva, J., Storms, Z. and Sauvageau, D. (2016) Host receptors for bacteriophage adsorption. FEMS Microbiol Lett. 363

53 Petersen, J., Heitz, M. J. and Hagan, I. M. (1998) Conjugation in S. pombe:

identification of a microtubule-organising centre, a requirement for microtubules and a role for Mad2. Curr Biol. 8, 963-966

54 Read, E. B., Okamura, H. H. and Drubin, D. G. (1992) Actin- and tubulindependent functions during Saccharomyces cerevisiae mating projection formation. Mol Biol Cell. 3, 429-444

55 Naor, A., Lapierre, P., Mevarech, M., Papke, R. T. and Gophna, U. (2012) Low species barriers in halophilic archaea and the formation of recombinant hybrids. Curr Biol. 22, 1444-1448

56 Yutin, N. and Koonin, E. V. (2012) Archaeal origin of tubulin. Biol Direct. 7, 10 57 Ettema, T. J. and Bernander, R. (2009) Cell division and the ESCRT complex: A surprise from the archaea. Commun Integr Biol. 2, 86-88

58 Makarova, K. S., Yutin, N., Bell, S. D. and Koonin, E. V. (2010) Evolution of diverse cell division and vesicle formation systems in Archaea. Nat Rev Microbiol. 8, 731-741

59 Pelve, E. A., Lindas, A. C., Martens-Habbena, W., de la Torre, J. R., Stahl, D. A. and Bernander, R. (2011) Cdv-based cell division and cell cycle organization in the thaumarchaeon Nitrosopumilus maritimus. Mol Microbiol. 82, 555-566

60 Ng, K. H., Srinivas, V., Srinivasan, R. and Balasubramanian, M. (2013) The Nitrosopumilus maritimus CdvB, but not FtsZ, assembles into polymers. Archaea. 2013, 104147

61 Makarova, K. S. and Koonin, E. V. (2010) Two new families of the FtsZ-tubulin protein superfamily implicated in membrane remodeling in diverse bacteria and archaea. Biol Direct. 5, 33

62 Stoeckenius, W. (1981) Walsby's square bacterium: fine structure of an orthogonal procaryote. J Bacteriol. 148, 352-360

63 Haeusser, D. P. and Margolin, W. (2016) Splitsville: structural and functional insights into the dynamic bacterial Z ring. Nat Rev Microbiol. 14, 305-319

64 Hamoen, L. W., Meile, J. C., de Jong, W., Noirot, P. and Errington, J. (2006) SepF, a novel FtsZ-interacting protein required for a late step in cell division. Mol Microbiol. 59, 989-999

65 Pohlschroder, M. and Albers, S. V. (2015) Editorial: Archaeal Cell Envelope and Surface Structures. Front Microbiol. 6, 1515

66 Pum, D., Messner, P. and Sleytr, U. B. (1991) Role of the S layer in morphogenesis and cell division of the archaebacterium Methanocorpusculum sinense. J Bacteriol. 173, 6865-6873

67 Typas, A., Banzhaf, M., Gross, C. A. and Vollmer, W. (2011) From the regulation of peptidoglycan synthesis to bacterial growth and morphology. Nat Rev Microbiol. 10, 123-136

68 Schoenemann, K. M. and Margolin, W. (2017) Bacterial Division: FtsZ Treadmills to Build a Beautiful Wall. Curr Biol. 27, R301-r303 


\section{Figure Legends}

Figure 1. The polymerization-depolymerization cycle of tubulin and the dynamic behavior of the microtubule. Tubulin superfamily proteins share a common fold and mode of GTP-dependent polymerization, with rare exceptions. They self-assemble (polymerize) into single-subunit thick "protofilaments", in which GTP molecules are positioned between successive subunits that are arranged in a head-to-tail manner. GTP hydrolysis requires both flanking subunits in the polymer, which destabilizes the polymers and promotes disassembly and turnover. The cycle of polymerization-depolymerization is regulated and provides the basis for assembly of a flexible and dynamic scaffold that drives structural changes or supports internal movement in essential cellular functions, such as DNA segregation and cell division. In microtubules, the stable $\alpha / \beta$-tubulin heterodimers (light/dark grey spheres) assemble longitudinally to form a protofilament, with beta-tubulin subunits facing the plus end and the alpha-tubulin subunits facing the minus end. The 13 protofilaments associate laterally in a hollow cylindrical arrangement to form the microtubule. Microtubules and other tubulinsuperfamily proteins are often highly dynamic, exhibiting growth and shrinkage (dynamic instability and treadmilling-like behaviors). By contrast, FtsZ proteins assemble from monomers and do not form microtubules, however, lateral associations between protofilaments are also important.

Figure 2. H. volcanii as a model for cell biology: localization of tubulin-superfamily proteins by live cell microscopy. (a) Use of fluorescent proteins as cytological markers (phase contrast and fluorescence image overlay); Mid-cell and division-site localization of $H$. volcanii FtsZ1-GFP occurs in essentially all cells in the population (the same results can be obtained with other fluorescent proteins [4]. As shown in some cells, division sometimes shows asymmetries, including noticeable deviation from mid-cell positioning, as well as asymmetric closure from one side of the cell. Z-rings frequently appear as discontinuous structures and show filaments or localizations that are somewhat misaligned with the ring. (b) Cryo-electron tomography slice shows high resolution detail of an elongated $H$. volcanii cell, with the characteristic pleated appearance of the archaeal S-layer and underlying cytoplasmic membrane (CM) visible. (c) Wild-type $H$. volcanii under Differential Interference Contrast (DIC) microscopy, and a mutant strain expressing a GTPase mutant of FtsZ1 that displays a gross cell division defect (ongoing cell growth without division), manifesting as giant cells that 
display a wrinkled envelope (mounted on gel pads). (d) Live-cell time-lapse microscopy showing microtubule-like dynamic instability (growth and retraction) during high-level overproduction of CetZ1-GFP. The phenomena is expected to be a non-physiological consequence of overproduction. The lower series shows in panel (d) shows a filament kinking and re-straightening event. (10-min frame intervals in both series - left to right). Scale bar $=2$ $\mu \mathrm{m}$. (e) Moderate supplementary expression of CetZ1-GFP, imaged during the transition of a plate shaped cell to a rod shaped cell, reveals patchy filaments and other localizations that are transient and frequently aligned with the rod-cell axis (15 min frame intervals). Scale bar $=1$ $\mu \mathrm{m}$. All data after ref [4].

Figure 3. Molecular organization and structure of the tubulin superfamily. (a) Domain organization and comparisom between the three principle families: tubulin, FtsZ and CetZ. The common colours represent approximate regions of the primary sequences that show high structural homology (yet often low sequence homology), and correspond to the colours given to the domains shown in (b) - the crystal structure of the CetZ2 protofilament from $H$. volcanii (PDB code 4B45). The termini show the greatest divergence in length and sequence and yet contain regions of critical importance for the function and specificity of interactions with other components of the cell. FtsZ proteins frequently display an N-terminal extension that is lacking in tubulins and CetZ proteins, and may be largely unfolded or display a helical segment (pink), and is of relatively poorly characterized function,. FtsZ also shows an unstructured C-terminal tail of variable length in different species, whereas tubulin and CetZ proteins show nonhomologous alpha-helical extensions (cyan). All three families exhibit different peptide motifs at the extreme C-termini that are typically used as a hub for the specific binding of numerous different cellular components or proteins that act on the cytoskeleton.

Figure 4. Assembly and dynamic behavior of FtsZ in cell division. (a) FtsZ monomer (gray sphere, left) has a globular GTPase fold, consisting of N-terminal and C-terminal domains with $\mathrm{N}$ - and C-terminal extensions that mediate interactions with other components (above). In the presence of GTP, FtsZ monomers polymerize longitudinally into protofilaments that associate with each other laterally to form filament bundles that further assemble to form the Z-ring (numerous other components not shown). In the Z-ring, FtsZ filaments show dynamic treadmilling (polymerizing at one end, while de-polymerizing at the other - see expansion). In Bacteria, FtsZ rings are tethered to the cytoplasmic membrane through several proteins 
including FtsA, ZipA (Gram-negative), or SepF (Gram-positive), whereas in Archaea only SepF has been clearly identified as a homolog, but its function is currently unknown). (b) The bacterial envelope (only Gram-negative model shown here) differs substantially compared to archaea (c). OM - outer membrane, PG - peptidoglycan, IM - inner membrane, CM cytoplasmic membrane. A Gram-positive and Gram-negative bacteria have a fundamentally similar FtsZ-based division mechanism that drives bacterial cell wall synthesis, which FtsZ dynamic treadmilling organizes peptidoglycan (PG) remodeling at mid-cell during division. Gram-negative species also need to coordinate outer membrane invagination with the constricting inner membrane and septum, aided by the Tol-Pal complex [67, 68]. The divisome of gram-negative species contains PG synthases PBP1B and PBP3, amidase enzymes (Ami), as well as Tol-Pal complex $[67,68]$. These complexes can move in either direction around the ring circumference to synthesize new layers of septal PG. (c) Hypothetical model of archaeal division fundamentals, whereby analogy to the bacterial system, FtsZ filament dynamics direct S-layer synthesis by recruitment of the Sec (secretion) and Agl (glycosylation) protein complexes at mid-cell. The lack of a PG wall and presence of a non-covalent paracrystalline S-layer glycoprotein mono-layer, external to the cytoplasmic membrane, suggests that division in archaea cannot work solely by directional ingrowth as is possible in bacteria, and might have a greater reliance on membrane constriction to direct the inward invagination of the division furrow.

\section{Acknowledgements}

This work was supported by the Australian Research Council (ARC DP160101076 and FT160100010). The authors declare that they have no competing interests. 
Figure 1.

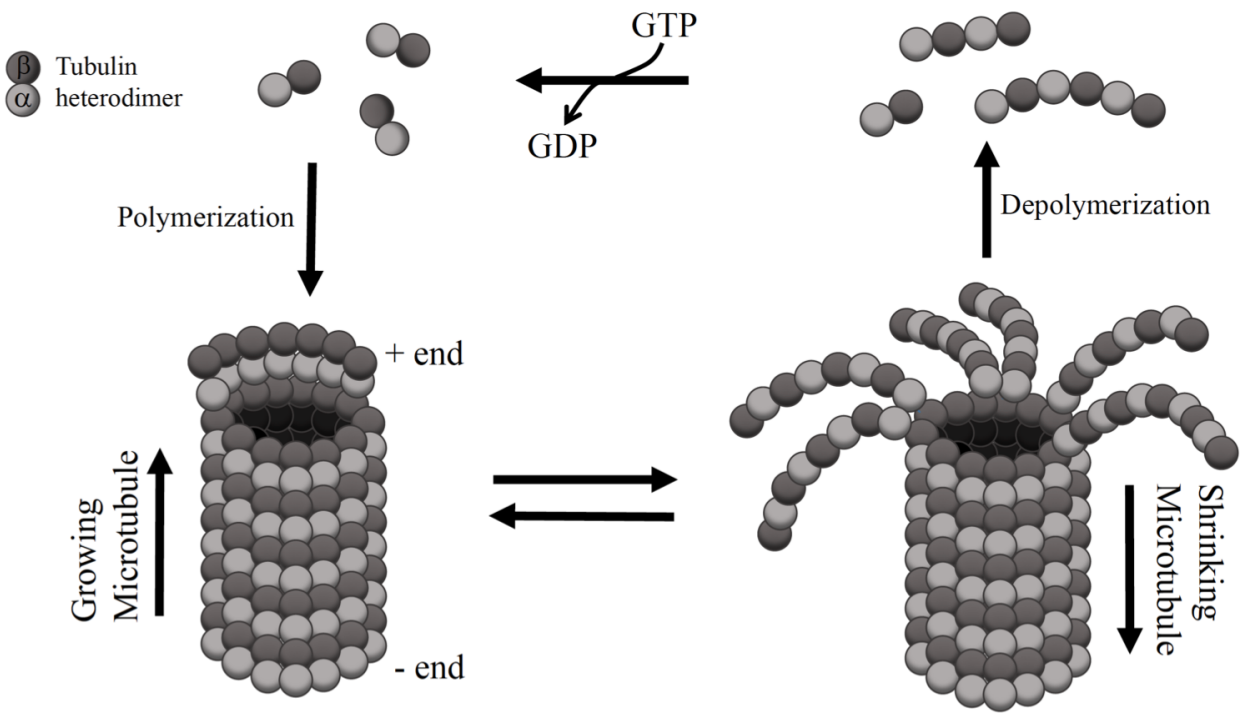


Figure 2

(a)

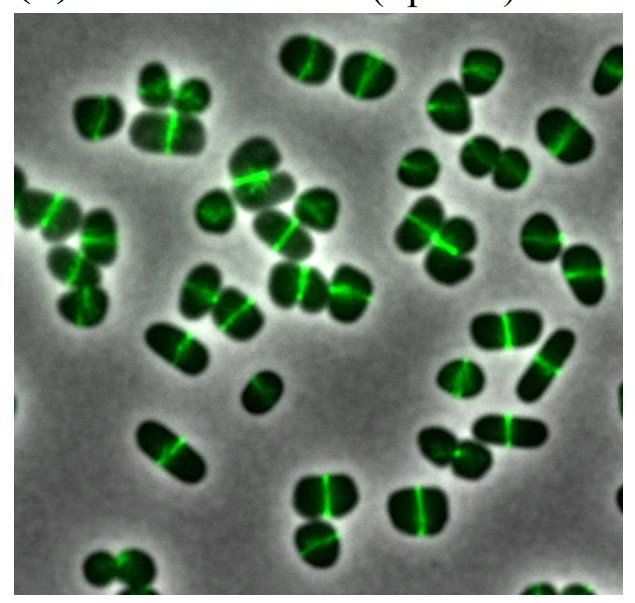

(c) Wild-type (DIC)

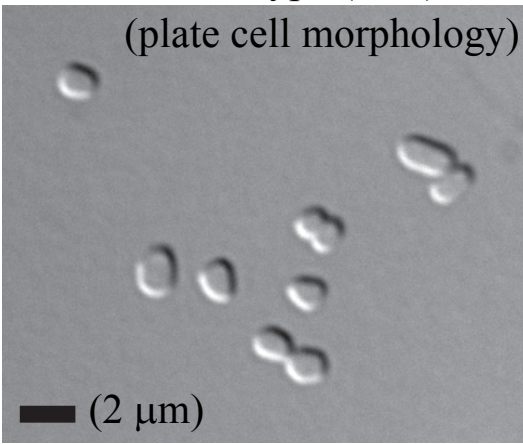

(b) CryoEM

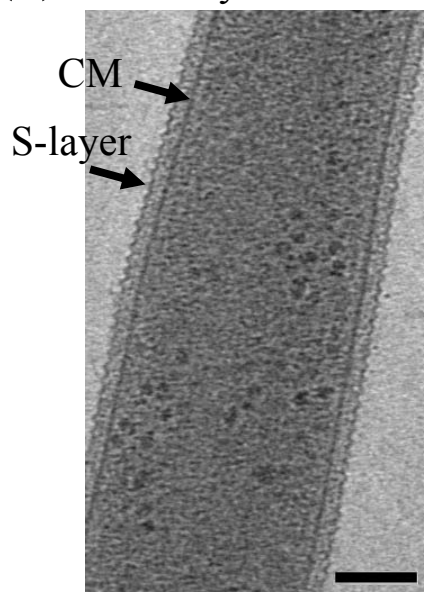

FtsZ1.D250A mutant

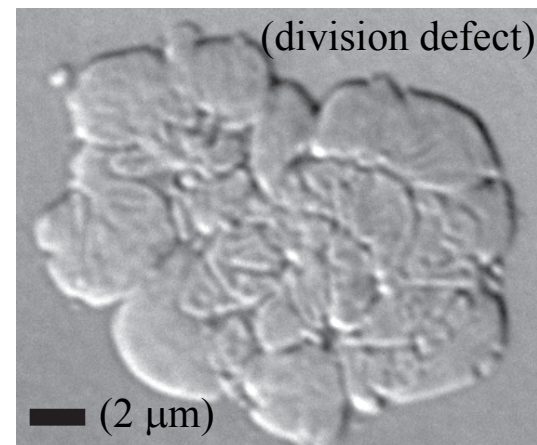

(d) CetZ1-GFP overproduction - dynamic instability

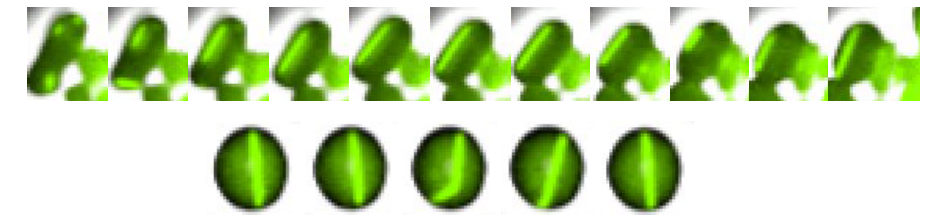

(e) CetZ1-GFP - moderate expression during rod-cell development

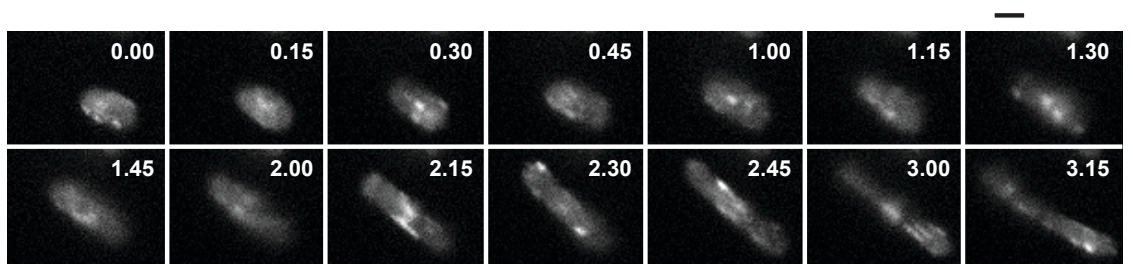




\section{Figure 3.}

(a) Tubulin/FtsZ/CetZ superfamily - domain structural similarity

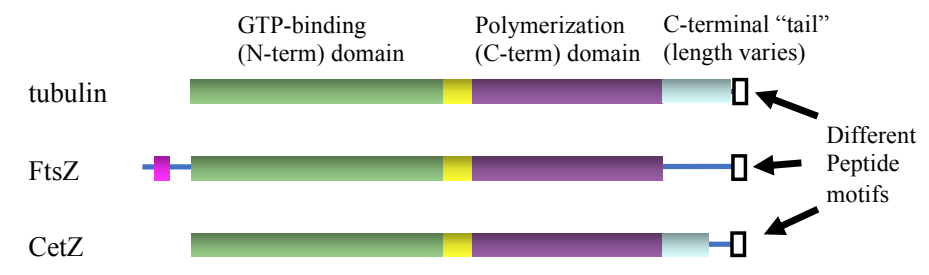

(b) CetZ2 protofilament

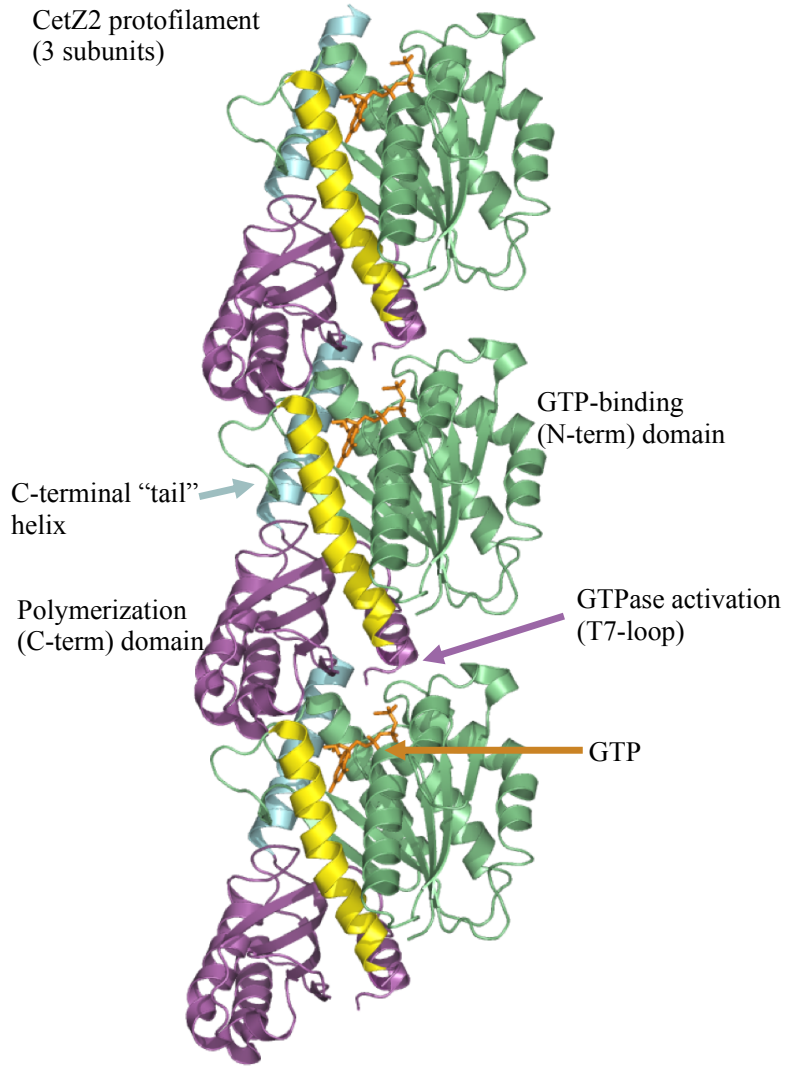




\section{Figure 4.}

(a)

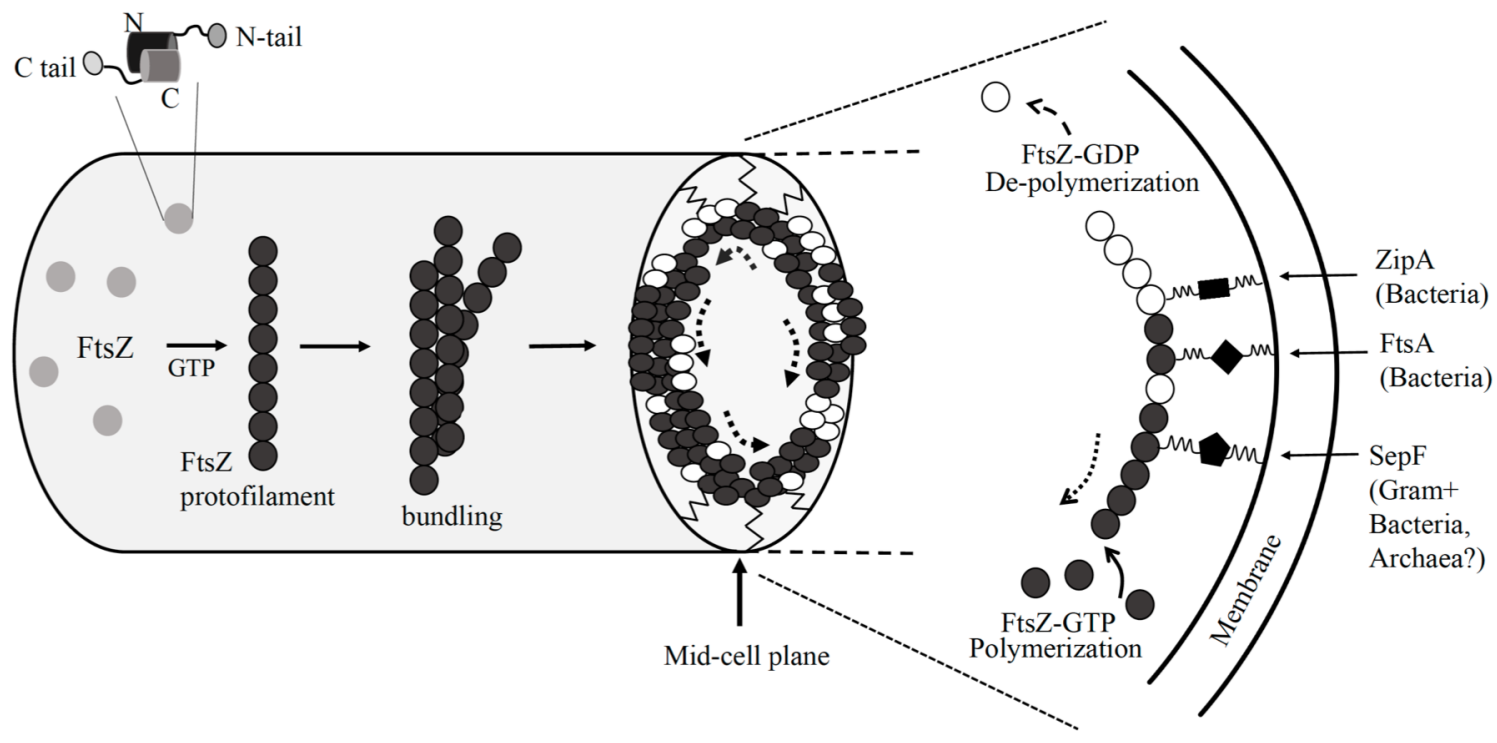

(b) Gram-negative bacteria

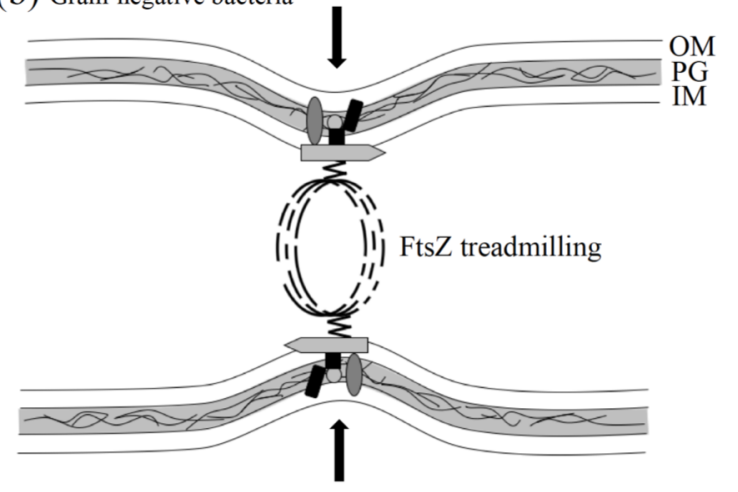

(c) Archaea

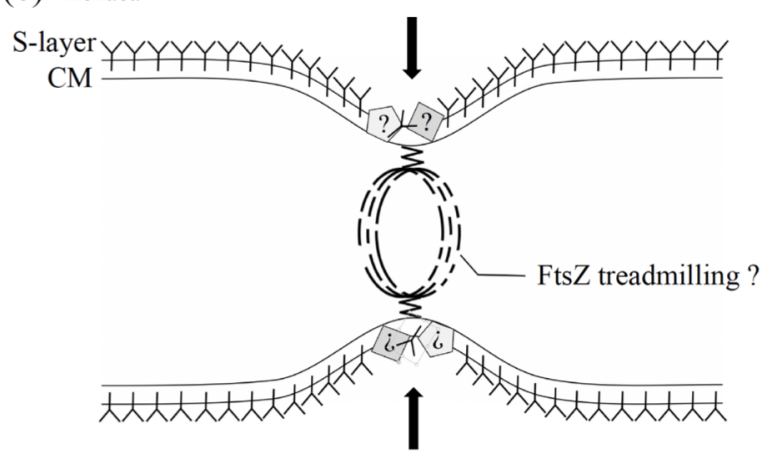

\begin{tabular}{llll}
0 & Tol-Pal & Y & Glycoprotein \\
PG synthases PBP1B & $\checkmark$ & Sec proteins \\
PG synthases PBP3 & $\diamond$ & Agl proteins \\
$\bigcirc$ & Ami & \\
$\square$ & IM-localized \\
cell division protein & \\
\hline
\end{tabular}

